\title{
Manejo Sustentável do Barbatimão no Norte de Minas Gerais
}

\author{
Messulan Rodrigues Meira ${ }^{1}$, Christian Dias Cabacinha ${ }^{2}$ \\ ${ }^{1}$ Programa de Pós-graduação em Ciências Agrárias, Instituto de Ciências Agrárias, Universidade Federal de \\ Minas Gerais - UFMG, Montes Claros/MG, Brasil \\ ${ }^{2}$ Instituto de Ciências Agrárias, Universidade Federal de Minas Gerais - UFMG, Montes Claros/MG, Brasil
}

\begin{abstract}
RESUMO
Neste estudo objetivou-se avaliar o manejo para a espécie Stryphondendron adstringens a partir de diferentes intensidades de cortes seletivos com controle por classe de diâmetro no norte de Minas Gerais. A produção estimada para elaboração dos planos foi obtida a partir de equações para peso seco de casca e volume de madeira para uma área de 31,28 ha e considerando uma demanda comercial de $7.000 \mathrm{~kg}$. ano ${ }^{-1}$ de cascas. De posse da produção foram simulados dez planos de manejo pelo método GDq. Os planos foram compostos pela remoção de percentuais de área basal (G), em múltiplos de dez a partir de $10 \%$ de remoção de $\mathrm{G}$, até o corte raso, totalizando dez planos. Os resultados obtidos mostraram que a colheita sustentável deve ser realizada dentro do intervalo de 40 a $70 \%$ de remoção de $\mathrm{G}$, que apresentaram respectivamente as seguintes produções de cascas e volume: $224,00 \mathrm{~kg}-\mathrm{ha}^{-1}$ e $367,16 \mathrm{~kg}-\mathrm{ha}^{-1} ; 0,63 \mathrm{~m}^{3}-\mathrm{ha}^{-1}$ e $1,06 \mathrm{~m}^{3}-\mathrm{ha}^{-1}$. Conclui-se que o manejo sustentável do barbatimão no norte de Minas Gerais é viável dentro dos intervalos de remoção de 40 a 70\% de área basal a partir do controle da colheita pelo método GDq.
\end{abstract}

Palavras-chave: Stryphnodendtron adstringens, cerrado, sustentabilidade, planta medicinal.

\section{Sustainable Management of Barbatimão in the Northern Minas Gerais State}

\begin{abstract}
This study aimed to evaluate the management for the Stryphnodendron adstringens species from different intensities of selective cuts with diameter class control in the northern Minas Gerais state. The estimated production for preparation of the plans was obtained from equation for dry weight of bark $(\mathrm{kg})$ and timber volume $\left(\mathrm{m}^{3}\right)$ for an area of $31.28 \mathrm{ha}$ and considering a commercial demand of the 7,000 kg.ha-1 of the barks. Ownership production were simulated ten management by GDq method. The plans were in multiples of ten from $10 \%$ removal G, up to clear cutting, totaling ten plans.

The results indicated that the sustainable haverst should be carried within the range of 40 to $70 \%$ removal of basal area, which respectively showed the following productions backs and volume: 224.00 and $367.16 \mathrm{~kg} \cdot \mathrm{ha}^{-1} ; 0.63 \mathrm{~m}^{3} \cdot \mathrm{ha}^{-1}$ and $1.06 \mathrm{~m}^{3} . \mathrm{ha}^{-1}$. Therewith we conclude that the sustainable management of barbatimão in the northern Minas Gerais state is viable within the removal ranges from 40 to $70 \%$ of basal area starting from the control of the havest by GDq method.
\end{abstract}

Keywords: Stryphnodendron adstringens, savanna, sustainability, medicinal plant. 


\section{INTRODUÇÃO}

O barbatimão [Stryphnodendron adstringens (Mart.) Coville], leguminosa da família Fabaceae, é uma das espécies arbóreas úteis do cerrado brasileiro. De valor econômico devido à produção eficiente de taninos nas cascas, já possui espaço consolidado no mercado, porém a sua exploração é totalmente extrativista predatória. Com a intensificação das coletas, essas atividades levaram a espécie à lista das ameaçadas de extinção (Borges \& Felfili, 2003). A perda de seus recursos genéticos já está refletindo de forma negativa, pois, atualmente, os extrativistas reclamam da dificuldade na aquisição da matéria-prima para a própria substistência (Gonçalves et al., 2012).

A maneira de conversão do quadro de predatório para sustentável ocorre a partir do manejo sustentável da espécie. Essa opção parte da capacidade de exploração das terras que possuem vegetação nativa com o barbatimão, a partir de estratégias que assegurem o uso sustentável dos recursos florestais. A mudança no hábito de exploração é possível pelo sistema silvicultural de cortes seletivos. Esse sistema utiliza o conceito de floresta balanceada, mantendo a estrutura inequiânea da floresta remanescente (Scolforo, 1998a, Scolforo et al., 2011). O sistema imita o processo de mortalidade natural para apropriar-se de estoque dos recursos e dinamizar a sucessão florestal (Souza \& Souza, 2005).

Esse sistema é baseado na área basal (G), no diâmetro máximo (D) e no quociente $q$ de De Liocourt (Rangel et al., 2006). Conhecido também como abordagem GDq, que está em conformidade com o artigo $2^{\circ}$ da Lei Estadual 10.561, de 27 de dezembro de 1991 (Minas Gerais, 1991), que diz que as atividades florestais deverão assegurar a manutenção da qualidade de vida e do equilíbrio ecológico e a preservação do patrimônio genético, de forma que o uso sustentado dos recursos preserve a biodiversidade.

Visando ao atendimento de questões legais para a comercialização da casca do barbatimão e à demanda de produção exigida pelo mercado consumidor no norte de Minas Gerais, objetivou-se avaliar planos de manejo para a espécie Stryphondendron adstringens a partir de diferentes intensidades de cortes seletivos com controle por classe de diâmetro no norte de Minas Gerais. Especificamente, buscou-se definir as melhores opções de manejo para atender ao mercado de cascas, levando em consideração a sustentabilidade da ação e respeitando os critérios legais para a colheita de casca e também de madeira para espécie em questão.

\section{MATERIAL E MÉTODOS}

Esta pesquisa foi realizada na Fazenda Bela Vista, no município de Botumirim, no norte do Estado de Minas Gerais. A área estudada é de cerrado stricto sensu, localizada próxima à sede da fazenda, nas seguintes coordenadas geográficas: latitude: $16^{\circ} 96^{\prime} 82^{\prime \prime} \mathrm{Sul}$, Longitude $43^{\circ} 0^{\prime} 75^{\prime \prime}$ Oeste e $892 \mathrm{~m}$ de altitude. A fazenda é uma propriedade particular, de 170 ha e possui solos com textura média e alto teor de alumínio com $\mathrm{Al}=1$, $3004 \mathrm{cmol} \mathrm{dm}^{-3}$ conforme análise físico-química recomendada pela $5^{\text {a }}$ aproximação (Ribeiro et al., 1999).

A área de estudo é de 31,28 ha, caracteriza bem a realidade das populações da espécie Stryphnodendron adstringens. É uma área em processo de regeneração avançado, sem a presença de animais domésticos. Nesta área foi realizado um inventário florestal onde foram distribuídas 25 parcelas de $20 \times 20 \mathrm{~m}\left(400 \mathrm{~m}^{2}\right)$, correspondendo a 1 ha amostrado, em transecções equidistantes a $80 \mathrm{~m}$. Cada extremidade foi marcada com estacas de tubos de PVC de $1 \frac{1}{2}$ ", enumerados para permitir a identificação (Souza et al., 2007).

Dentro de cada parcela foram mensurados todos os indivíduos adultos vivos com diâmetros a 1,30 m do solo (DAP) $\geq 3 \mathrm{~cm}$ com auxílio de uma suta (Costa et al., 2010). A distribuição dos diâmetros foi obtida a partir da fórmula de Spiegel: IC = A/nc; Onde $\mathrm{A}=$ amplitude, $\mathrm{nc}=$ número de classes, sendo que $\mathrm{nc}=1+3,3 \log (\mathrm{n})$ e $\mathrm{n}=$ número de individuos. Após obtenção da frequência, obteve-se o quociente q de De Liocourt que é a razão do número de árvores entre as classes de diâmetro sucessivas a partir da fórmula: $\mathrm{q}=\mathrm{Ni} / \mathrm{Ni}+1$, em que: $\mathrm{Ni}=$ número de árvores da iésima classe de diâmetro e $\mathrm{Ni}+1=$ número de árvores da iésima mais uma classe de diâmetro subsequente (Felfili \& Rezende, 2003). Determinada a distribuição diamétrica, os dados de frequência $\left(\mathrm{y}_{\mathrm{i}}\right)$ foram ajustados a partir do valor central das classes $\left(\mathrm{x}_{\mathrm{i}}\right)$ ao modelo de Meyer $\left(\operatorname{Lny}_{\mathrm{i}}=\beta_{0}+\beta_{1}^{*} \operatorname{Lnx} \mathrm{i}_{\mathrm{i}}\right)$ para verificar se a distribuição se ajustava a uma curva exponencial negativa conforme o padrão esperado nessa distribuição para espécie nativa (Scolforo, 1998b).

Com base na distribuição diamétrica obtida pelo inventário florestal, foi possível simular a intensidade de corte por meio da área basal total. Para a produção 
de casca e madeira, utilizaram-se as equações desenvolvidas por Gama (2012) para a produção da Stryphnodendron adstringens na área do estudo, Equação 1: LnPsc = -3,71840864 + 0,89619031 Ln (dap²ht) paraopesosecodecasca $(\mathrm{kg})$,comcoeficientededeterminação $\left(\mathrm{R}^{2}\right)$ e erro padrão residual (Syx) de 96,67\% e $\pm 26,45 \%$ e Equação 2: LnV = -10,1745813 + 1,005891616 Ln (dapht), para o volume de madeira $\left(\mathrm{m}^{3}\right)$, com coeficiente de determinação $\left(\mathrm{R}^{2}\right)$ e erro padrão residual (Syx) de $97,25 \%$ e $\pm 27,87 \%$.

Após estudo preliminar dos dados, foram definidos parâmetros para avaliar a intensidade dos corte para o manejo para a espécie. Adotou-se como alternativa de manejo o sistema de corte seletivo com controle por classe diamétrica, que consiste na divisão da área em talhões, de acordo com o tempo necessário para a regeneração da vegetação previsto em legislação. O ciclo de corte foi de dez anos, conforme a Portaria IEF n 054, de 25/08/1997 (Minas Gerais, 1997), para a reposição da área basal para o Cerrado (Figueiredo et al., 2010, Oliveira, 2006). Com base nesse horizonte de planejamento, a área permitida para remoção é de um talhão por ano, de forma aleatória. Esse sistema é bem empregado em solos com as características físicas e químicas da região estudada, por ser menos produtivo e pedregoso, com terreno acidentado inóspito para a agricultura, onde as árvores nativas não atingem grandes dimensões (Scolforo, 1998a).

O manejo foi definido, de forma a respeitar os mecanismos de sustentação do ecossistema conforme prescrito pelo Decreto Federal no 1282, de 1994, normatizado pelo artigo 15 da Lei 4771 de 15 de setembro de 1965, (Brasil, 1994) por meio de uma abordagem GDq. Nesta pesquisa a abordagem GDq foi modificada, onde o estoque da floresta foi controlado, baseando-se apenas na remoção de área basal do sítio. O diâmetro máximo (D) e o quociente $q$ de De Liocourt não sofreram alterações no seu valor original (Rangel et al., 2006, Scolforo, 1998a, Scolforo et al., 2011).

Os cortes simulados dentro desse sistema foram realizados em múltiplos de dez, a partir de $10 \%$ de remoção de G, sendo: $10 \%, 20 \%, 30 \%, 40 \%, 50 \%$, $60 \%, 70 \%, 80 \%, 90 \%$ e $100 \%$, totalizando dez planos. As intervenções de colheita foram simuladas a partir da equação obtida pelo modelo proposto por Meyer em sua forma linearizada, respeitando-se o conceito de floresta balanceada, como segue: $\operatorname{Lnyy}=5,430569-0,26156^{*} \operatorname{Ln} x_{i}$,
$R^{2}=92,81 \%$ e Syx $= \pm 0,4050$. Em que: $y i=$ frequência esperada; $\mathrm{xi}$ = valor central da classe diamétrica.

Nesse modelo, a remoção dos indivíduos por classe diamétrica tende a manter a estrutura do povoamento em exponencial negativo, que é o comportamento esperado para estrutura de florestas inequiâneas. Com base no modelo ajustado para a estrutura original da população, calcularam-se novos parâmetros para a equação ajustada considerando-se o percentual de área basal remanescente.

O número de árvores por classe diamétrica a ser removido para cada plano simulado foi obtido subtraindo a frequência remanescente, da original. O critério utilizado para determinar o manejo sustentável foi com base na capacidade da área de estudo em atender a demanda de produção anual de cascas em 7.000 kg.ano ${ }^{-1}$, ou seja, 583,3 kg.mês ${ }^{-1}$. Também comparou-se a produção de cascas dos diferentes planos simulados com a produção obtida no corte raso e nos fatores ecológicos e ambientais. Neste estudo, os planos selecionados como mais viáveis foram aqueles que apresentaram produção máxima, mantendo percentuais de área basal suficiente para promover a resiliência da área sob intervenção.

\section{RESULTADOS E DISCUSSÃO}

De acordo com o inventário realizado, a distribuição diamétrica da população comportou-se como o previsto para florestas inequiâneas, em razão de apresentar curva de distribuição diamétrica, assemelhando-se a um "J-reverso", evidenciando, que em florestas nativas, realmente há essa tendência do balanceamento entre mortalidade e recrutamento (Figura 1) (Scolforo, 1998a, Meyer, 1952).

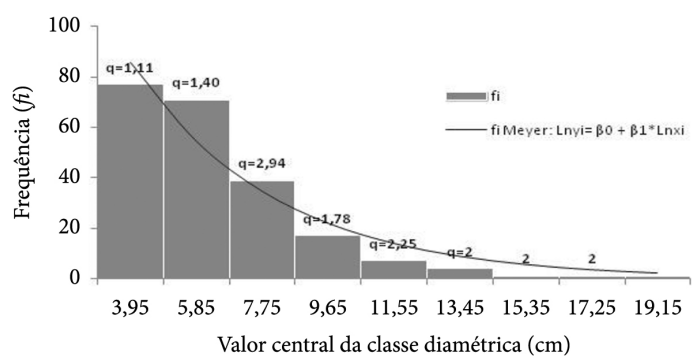

Figura 1. Histograma de frequência da distribuição horizontal em classes de diâmetro.

Figure 1. Frequency histogram of horizontal distribuition diameter classes of area studied. 
A curva ajustada a partir da equação de Meyer em sua forma linearizada confirmou esse comportamento, onde o número de árvores diminui exponencialmente nas classes de tamanho crescente. Para avaliar os planos de manejo, buscou-se analisar o balanceamento da estrutura removida quando confrontada com a original, a integridade da população e a demanda de mercado. A Tabela 1 apresenta a frequência de indivíduos observada (Fo) no inventário florestal, o peso seco $(\mathrm{kg})$ de cascas e o volume $\left(\mathrm{m}^{3}\right)$ da madeira para as diferentes classes diamétricas.

Na simulação feita para a remoção de 10, 20, 30, 40, 50 e 60\% da área basal, observou-se que a remoção de indivíduos está concentrada nas menores classes de diâmetro, seguindo o padrão natural de distribuição de indivíduos para manter a estrutura balanceada. Também os planos com menores remoções de área basal apresentaram maior déficit de indivíduos na estrutura removida nas maiores classes diamétricas. Nesse caso, as classes com essa deficiência não podem sofrer intervenção (Tabela 2). O déficit de indivíduos na classe $8^{\mathrm{a}}(16,65-18,60 \mathrm{~cm})$, não foi considerado para análise, uma vez que, esta classe apresentou ausência de indivíduos para a distribuição diamétrica original conforme apresentado na frequência observada na Tabela 1.

Nesse caso, indivíduos pertencentes à $5^{\mathrm{a}}, 6^{\mathrm{a}}, 7^{\mathrm{a}} \mathrm{e} 9^{\mathrm{a}}$ classes não podem sofrer intervenção, devido à ausência de representantes. No plano com $10 \%$ de remoção de $\mathrm{G}$ ), o corte pode ser feito até a $4^{\text {a }}$ classe. Para os planos

Tabela 1. Estrutura original da espécie Stryphnodendron adstringens na área de estudo.

Table 1. Original strutural of Stryphnodendron adstringens specie in the study area.

\begin{tabular}{|c|c|c|c|c|}
\hline \multirow{2}{*}{$\begin{array}{l}\text { Ordem das classes } \\
\text { diamétricas }\end{array}$} & \multirow{2}{*}{$\begin{array}{l}\text { Classes diamétricas } \\
(\mathbf{c m})\end{array}$} & \multicolumn{3}{|c|}{ Estrutura original } \\
\hline & & Fo & PS. casca $\mathrm{m}^{3} / \mathrm{ha}^{-1}$ & V. madeira kg/ha ${ }^{-1}$ \\
\hline $1^{\mathrm{a}}$ & $3,00--\mid 4,95$ & 73 & 39,66 & 0,0939 \\
\hline $2^{\mathrm{a}}$ & 4,95- - 6,90 & 66 & 98,44 & 0,2575 \\
\hline $3^{\mathrm{a}}$ & $6,90--\mid 8,85$ & 47 & 135,33 & 0,3831 \\
\hline $4^{\mathrm{a}}$ & $8,85--\mid 10,80$ & 16 & 81,69 & 0,2482 \\
\hline $5^{\mathrm{a}}$ & $10,80--12,75$ & 9 & 69,69 & 0,2222 \\
\hline $6^{a}$ & $12,75--\mid 14,70$ & 4 & 42,23 & 0,1401 \\
\hline $7^{\mathrm{a}}$ & $14,70--\mid 16,65$ & 2 & 32,25 & 0,1123 \\
\hline $8^{\mathrm{a}}$ & $16,65--\mid 18,60$ & 0 & 0,00 & 0,0000 \\
\hline \multirow[t]{2}{*}{$9^{a}$} & $18,60--\mid 20,55$ & 1 & 10,99 & 0,0365 \\
\hline & Total & 218 & 510,32 & 1,4939 \\
\hline
\end{tabular}

Fo = Frequência observada; PS. = Peso seco; V. = Volume

Tabela 2. Número de indivíduos a serem removidos por classe de diâmetro da Stryphnodendron adstringens para os diferentes percentuais de área basal $(\mathrm{G})$.

Table 2. Individuals number to be removed by the Stryphnodendron adstringens diameter class for different percentages of basal area $(G)$.

\begin{tabular}{|c|c|c|c|c|c|c|c|c|c|c|c|}
\hline \multirow{2}{*}{$\begin{array}{l}\text { Ordem das } \\
\text { classes diamé- } \\
\text { tricas }\end{array}$} & \multirow{2}{*}{$\begin{array}{l}\text { Classes diamé- } \\
\text { tricas }(\mathrm{cm})\end{array}$} & \multicolumn{10}{|c|}{ Percentual de remoção de G } \\
\hline & & $10 \%$ & $20 \%$ & $30 \%$ & $40 \%$ & $50 \%$ & $60 \%$ & $70 \%$ & $80 \%$ & $90 \%$ & $100 \%$ \\
\hline $1^{\mathrm{a}}$ & $3,00--\mid 4,95$ & 8 & 15 & 23 & 30 & 37 & 44 & 51 & 59 & 66 & 73 \\
\hline $2^{\mathrm{a}}$ & $4,95--\mid 6,90$ & 27 & 31 & 35 & 40 & 44 & 48 & 53 & 57 & 62 & 66 \\
\hline $3^{\mathrm{a}}$ & $6,90--\mid 8,85$ & 23 & 26 & 28 & 31 & 34 & 36 & 39 & 42 & 44 & 47 \\
\hline $4^{\mathrm{a}}$ & $8,85--\mid 10,80$ & 1 & 3 & 5 & 6 & 8 & 10 & 11 & 13 & 14 & 16 \\
\hline $5^{\mathrm{a}}$ & $10,80--\mid 12,75$ & 0 & 1 & 2 & 3 & 4 & 5 & 6 & 7 & 8 & 9 \\
\hline $6^{a}$ & $12,75--\mid 14,70$ & -1 & -1 & 0 & 0 & 1 & 2 & 2 & 3 & 3 & 4 \\
\hline $7^{\mathrm{a}}$ & $14,70--\mid 16,65$ & -1 & -1 & -1 & 0 & 0 & 1 & 1 & 1 & 2 & 2 \\
\hline $8^{a}$ & $16,65--\mid 18,60$ & -2 & -2 & -2 & -1 & -1 & -1 & -1 & 0 & 0 & 0 \\
\hline $9^{a}$ & $18,60--\mid 20,55$ & 0 & 0 & 0 & 0 & 0 & 0 & 1 & 1 & 1 & 1 \\
\hline
\end{tabular}


20, 30 e 40\% de G, em relação ao anterior, reduziu-se uma classe com déficit de indivíduos, de forma que o corte seria permitido até a $5^{\text {a }}$ classe. Nos planos 50 e $60 \%$ de $\mathrm{G}$, também houve uma redução de uma classe com déficit, em que o corte pode ser feito até a $6^{\mathrm{a}}$ e $7^{\mathrm{a}}$ classe, respectivamente. A partir do plano $70 \%$ de remoção de $\mathrm{G}$, todas as classes foram representadas por indivíduos, não apresentando déficit de indivíduos em nenhuma das classes, com exceção da $8^{\mathrm{a}}$ classe, pelos motivos já citados.

O critério de seleção das melhores opções de manejo também levou em consideração o tamanho da área de estudo (31,28 ha) que foi dividida em dez talhões para simulação dos planos (Tabela 3).

De acordo os planos propostos, a área de produção decresce à medida que se aumenta o percentual de remoção de área basal como esperado, contudo para atender à exigência de produção, os três primeiros planos (10, 20 e $30 \%$ de G) não contemplam essa meta, pois esses requerem uma área superior à área estudada. A grande demanda de área exigida nessas opções, juntamente com a baixa produção, tornam-os insignificantes para justificar uma análise neste estudo.

Para atender à demanda comercial de cascas de $7.000 \mathrm{~kg} \cdot \mathrm{ano}^{-1}$, é necessário uma produção mínima de 583,3 kg.mês ${ }^{-1}$. Considerando os planos atendidos dentro da área de estudo, a produção efetiva por talhão varia de 700,67 a 1594,78 kg de cascas por ano, refente aos planos de 40 a $100 \%$ de G removido (Tabela 3). O período de fornecimento de casca de cada talhão varia de 36 a 82 dias atingindo de $10 \%$ a $22,78 \%$ da produção exigida pelo mercado (Tabela 4).

Ao comparar a produção de cascas obtida por hectare com a capacidade de produção total, observou-se que, proporcionalmente, as maiores produções estão concentradas nos planos de menor percentual de remoção. Por exemplo, o plano 40\% de remoção de G, gera uma produção de 3,94\% superior à produção do corte raso; no plano $50 \%$, é 3,15\% a mais; no de $60 \%$, é $2,73 \%$; no de $70 \%$, é $2,01 \%$; no de $80 \%$ e $1,37 \%$ e no de $90 \%$, é 0,73\%. Nos planos 70, 80 e $90 \%$ de remoção $\mathrm{G}$, mesmo com a frequência esperada positiva em todas as classes, a produção foi inferior aos menores percentuais de $\mathrm{G}$, em termos de produção equivalente ao corte raso (Tabelas 2 e 5).

Essa observação foi feita com base no potencial produtivo dos planos 40, 50 e 60\%, mesmo sob déficit de indivíduos nas maiores classes diamétricas. Considerando-se o déficit como o fator limitante para a produção, caso esse problema não existisse, a produção seria maior do que foi apresentada no resultado e os impactos causados na área seriam os mesmos. Entretanto, para as mesmas estruturas removidas apresentadas pelos cortes mencionados, é mais viável a colheita em

Tabela 3. Valores remanescente (reman.) e removida (remov.) para peso seco (PS) de casca/kg e volume (V) de madeira $/ \mathrm{m}^{3}$, produção obtida por talhão, e estimativa de área para atendimento da demanda comercial sob valor removido de área basal.

Table 3. Remaining values (reman.) and removed values (remov.) to dry weight (DW) shell/kg and amount (A) of $\mathrm{wood} / \mathrm{m}^{3}$, obtained by plot, and area stimation for demand reaching under commercial value of basal area removed.

\begin{tabular}{|c|c|c|c|c|c|c|c|}
\hline \multirow{2}{*}{$\begin{array}{l}\text { Remoção } \\
\text { de G (\%) }\end{array}$} & \multicolumn{2}{|c|}{$\begin{array}{c}\text { PS. de casca } \\
\left(\mathrm{kg}^{\left.-h^{-1}\right)}\right.\end{array}$} & \multicolumn{2}{|c|}{ V. de madeira $\left(\mathrm{m}^{3} \cdot \mathrm{ha}^{-1}\right)$} & \multicolumn{2}{|c|}{$\begin{array}{c}\text { Produção/UPA } \\
(3.128 \text { ha })\end{array}$} & \multirow{2}{*}{ DA } \\
\hline & Reman. & Remov. & Reman. & Remov. & $\begin{array}{c}\text { Casca } \\
(\mathrm{kg})\end{array}$ & $\begin{array}{c}\text { Madeira } \\
\left(\mathrm{m}^{3}\right)\end{array}$ & \\
\hline 10 & 429,47 & 80,84 & 1,295 & 0,1983 & 252,87 & 0,62 & 86,59 \\
\hline 20 & 381,75 & 128,57 & 1,151 & 0,3423 & 402,17 & 1,07 & 54,45 \\
\hline 30 & 334,03 & 176,29 & 1,007 & 0,4862 & 551,43 & 1,52 & 39,71 \\
\hline 40 & 286,31 & 224,00 & 0,863 & 0,630 & 700,67 & 1,97 & 31,25 \\
\hline 50 & 238,59 & 271,72 & 0,719 & 0,774 & 849,94 & 2,42 & 25,76 \\
\hline 60 & 190,88 & 319,44 & 0,575 & 0,918 & 999,21 & 2,87 & 21,91 \\
\hline 70 & 143,16 & 367,16 & 0,431 & 1,06 & $1.148,48$ & 3,32 & 19,07 \\
\hline 80 & 85,44 & 414,88 & 0,287 & 1,21 & 1.29774 & 3,78 & 16,87 \\
\hline 90 & 47,72 & 462,60 & 0,144 & 1,35 & $1.447,01$ & 4,22 & 15,13 \\
\hline 100 & 0,48 & 509,84 & 0,001 & 1,41 & $1.594,78$ & 4,41 & 13,73 \\
\hline
\end{tabular}

$\mathrm{G}=$ Área basal; $\mathrm{DA}=$ Demanda de área (ha) para produção de $7.000 \mathrm{~kg} \cdot \mathrm{ano}^{-1}$ de casca seca. 
Tabela 4. Período estimado em dias para a área atender a produção de cascas dentro da escala de produção.

Table 4. Estimated period of days to cater to production area within the barks scale production.

\begin{tabular}{ccc}
$\begin{array}{c}\text { Remoção } \\
\text { de G (\%) }\end{array}$ & $\begin{array}{c}\text { Tempo de } \\
\text { produção em dias } \\
\text { por talhão }\end{array}$ & $\begin{array}{c}\text { Percentual da } \\
\text { produção } \\
\text { atendida (\%) }\end{array}$ \\
\hline 40 & 36,03 & 10,00 \\
50 & 43,71 & 12,14 \\
\hline 60 & 46,30 & 14,27 \\
70 & 59,06 & 16,41 \\
\hline 80 & 66,74 & 18,53 \\
\hline 90 & 74,48 & 20,67 \\
\hline 100 & 82,02 & 22,78 \\
\hline
\end{tabular}

Tabela 5. Percentual de produção de cascas em função do corte raso.

Table 5. Percentage of production of barks in function of total cutting.

\begin{tabular}{ccc}
$\begin{array}{c}\text { Remoção de } \\
\text { G (\%) }\end{array}$ & $\begin{array}{c}\text { Peso seco } \\
\text { Kg-ha }^{-1}\end{array}$ & $\begin{array}{c}\text { Percentual de } \\
\text { produção equilavente } \\
\text { ao corte raso\% }\end{array}$ \\
\hline 40 & 224,00 & 43,94 \\
50 & 271,00 & 53,15 \\
\hline 60 & 319,44 & 62,73 \\
70 & 367,16 & 72,01 \\
\hline 80 & 414,88 & 81,37 \\
\hline 90 & 462,60 & 90,73 \\
\hline 100 & 509,84 & - \\
\hline
\end{tabular}

duas áreas de tamanhos iguais do que a exploração de uma única área sob o corte raso. A maior produção equivalente nos planos de menor remoção de $G$, pode estar associada ao fato das plantas jovens com menores diâmetros produzirem a maior quantidade de cascas (Camolesi, 2007, Rufini et al., 2010).

Dessa forma, diante da demanda em área, da produção de cascas e da sustentabilidade, os planos 40,50 e $60 \%$ de $\mathrm{G}$ foram considerados viáveis neste estudo. Embora a frequência esperada para a estrutura removida tenha apresentado classes diamétricas sem representantes, esse fator não impede sua aplicação, pois com o tempo, ocorrerá um balanceamento entre as classes diamétricas, com prováveis incrementos diamétricos que o manejo da vegetação promoverá (Souza \& Souza, 2005).

Em relação à análise de produção sob a remoção de 70, 80 e $90 \%$ de G, quando comparado ao corte raso, o de $70 \%$ de $\mathrm{G}$ se destacou dos demais. Esse plano mostrou que, em critérios conservacionistas, dentre todos que apresentaram superávit de indivíduos em todas as classes, foi o mais eficiente. Esse resultado corrobora o estudo de Souza et al. (2011), pois, esses autores ao realizarem estudos de corte em áreas de cerrado stricto sensu no município de Brasilândia-MG, no período de 1997 a 2008, verificaram que a remoção de área basal em 70\%, além de gerar maior produção equivalente a grande remoção de indivíduos jovens, também apresentou maior ganho em número de indivíduos por espécie quando comparado aos cortes mais severos.

O critério utilizado para a seleção do plano de $70 \%$ de $\mathrm{G}$, foi com base na manutenção do banco de sementes, pois sabe-se que as sementes do barbatimão possuem dureza de tegumento, dificultando a penetração da água. Sob condições naturais de abrigo para a regeneração, o índice de germinação é muito baixo, variando entre 4 a $12 \%$ o percentual de sementes germinadas (Côrrea et al., 2012, Martins et al., 2008). Não obstante esse não é o único motivo comprometedor da germinação. As sementes também são altamente predadas por insetos, tornando-as inviáveis física e fisiologicamente (Côrrea et al., 2012). Por isso, é necessário que o percentual de área basal removido escolhido permita que a vegetação remanescente abrigue as sementes. Segundo BCMF (1999), para planos de manejo em que a espécie necessita de umidade para romper o tegumento, como o caso específico do barbatimão, os níveis de área basal para o abrigo devem ter uma proporção igual ou superior a 30\% da área basal original.

Ainda que o corte raso (100\%) possua maior simplicidade e rapidez na execução, a colheita por esse sistema não garante a manutenção das populações em suas regiões de origem (Almeida et al., 2009). Esse fato foi verificado por Oliveira (2006), ao estudar o manejo do cerrado stricto sensu sob corte raso, durante o período de sete anos. Esse autor observou que não houve crescimento em diâmetro, comprometendo a recuperação da área nos níveis iniciais. Scolforo (1998b) recomendou a eliminação da possibilidade do manejo sob esse percentual mesmo deixando $10 \%$ de árvores porta sementes por classe diamétrica. A justificativa apresentada pelo autor foi que muitos fragmentos florestais, principalmente no Cerrado do Brasil Central, foram quase totalmente dizimados pelo emprego desse 
sistema. Para esse autor, esse plano só deve ser aplicado quando a floresta não responder satisfatoriamente a nenhum outro plano silvicultural. E ainda assim, deve ser manejado com plantio de enriquecimento e produção de mudas para reduzir o impacto provocado pela grande área de clareira aberta após o corte.

Diante de todas essas questões analisadas e da observação feita anteriormente acerca da produção equivalente de casca ser inversamente proporcional à remoção de área basal, definiu-se que o limite máximo de remoção de área basal deve ser de 70\% de G. Assim oferecem-se mais condições à população de se regenerar, por manter as árvores porta sementes transmissoras das características genéticas desejáveis para a próxima geração, tornando o plano de manejo mais sustentável (BCMF, 1999).

Então, além dos planos 40, 50 e $60 \%$ já definidos anteriormente, o plano $70 \%$ atendeu também às exigências de produção. Assim, a remoção de 40 até $70 \%$ de G são mais sustentáveis, uma vez que esses planos apresentaram os pré-requesitos básicos necessários a um sistema de manejo florestal: melhor eficiência de produção de cascas e madeira, com os menores impactos ambientais possíveis.

Apesar de mais de uma opção de corte ter apresentado-se viável, sugere-se que a colheita inicial sob o menor percentual de remoção de G seja utilizada, dentro do limite da área de estudo ( $40 \%$ de G), porque caso a demanda de casca aumente ao longo dos anos mais rápido do que a oferta, a produção contará com o próximo plano subsequente.

Ao comparar a madeira gerada com a quantidade de casca, observou-se que o volume de madeira é muito pequeno (Tabela 3). Como o foco principal do estudo foi avaliar o manejo do barbatimão para a produção de casca, a madeira foi considerada como subproduto da colheita florestal. Em outros trabalhos, sobretudo na Amazônia, a madeira é considerada como o produto principal, devido ao seu alto valor no mercado madeireiro, assim, não se pode desconsiderar o seu potencial. Caso a devida importância não seja atribuida ao produto e optar por deixá-lo abandonado no campo, o sítio ficará exposto a sérios danos ambientais (Faria, 2009), gerando material combustível, o que pode intensificar a ocorrência de incêndios, além de provocar a proliferação de pragas (Scolforo, 1998b). Mesmo quando o fogo é induzido de forma natural, a queima de dejetos madeireiros é algo desaconselhável, porque esses ampliam a dimensão do fogo, deixando de exercer a função ecológica como um evento natural e passam a atuar como uma catástrofe ambiental. Além desse fator, a madeira abandonada em campo, quando queimada, mineraliza rapidamente a matéria orgânica, transformando-a em cinzas minerais fertilizantes. Com isso, perdem-se a cobertura do solo e, na primeira chuva forte, perdem-se essas cinzas também, que serão arrastadas pelas enxurradas. A permanência do resíduo no campo é a pior opção, além de ser ilegal (Sano et al., 2008).

Como a madeira originada neste estudo é de baixa produção e está sujeita às adversidades edafoclimáticas e à instabilidade da oferta de mercado, essa precisa ter um destino definido. Para isso, a alternativa que se pode oferecer ao produtor florestal é a separação de toras de maior diâmetro, para destinar-se, à construção de cercas, pelo fato dessas apresentarem resistência à água e ao sol e possuírem estrutura bem lignificada (Almeida et al., 1998). Quanto à madeira com menores diâmetros, deve ser destinadas à geração de energia doméstica como lenha. Sabe-se que a produção de lenha é onerosa e o preço de madeira para energia é crescente, devido à escassez desse tipo de recurso oriundo de florestas naturais. Assim, o uso da lenha no Brasil para a cocção de alimentos pelas comunidades rurais é muito significativo. Acredita-se que 24,7\% da energia doméstica consumida no Brasil, o maior percentual é de lenha, representada por 10,6\% e, dentro desse percentual, $27,8 \%$ são de fontes naturais renováveis (BEN, 2012). Dessa forma, o manejo desse produto passa a ser interessante, por gerar renda para o produtor, ainda que o dono ou arrendatário do terreno opte por consumir a madeira produzida, ao em vez de vendê-la, essa teria um custo para o produtor, ao efetuar o pagamento pela colheita da mesma aos trabalhadores de campo (REMADE, 2004).

A baixa produção de madeira discutida anteriormente foi sob a capacidade de produção de um hectare, mas a madeira produzida como subproduto da colheita de $7.000 \mathrm{~kg}$ de casca, representa um volume considerável para a venda (Tabela 6).

Para a menor intensidade de corte, $10 \%$ de área basal removida, observou-se que a obtenção de 7 t.ha ${ }^{-1}$ de casca, há uma produção real média de lenha equivalente a $6,2 \mathrm{~m}^{3}$. Essa observação mostra que o volume de 
Tabela 6. Total de lenha gerada em função da quantidade de casca produzida para atender a demanda comercial de $7.000 \mathrm{~kg}$ de casca da Stryphnodendron adstringens.

Table 6. Total of firewood generated depending on the amount of bark produced to answer the commercial demand of $7.000 \mathrm{~kg}$ of bark Stryphnodendron adstringens.

\begin{tabular}{ccc|}
$\begin{array}{c}\text { Área basal } \\
\text { removida (\%) }\end{array}$ & $\begin{array}{c}\text { Madeira/ha } \\
\left(\mathbf{m}^{\mathbf{3}}\right)\end{array}$ & $\begin{array}{c}\text { PMG } \\
\left(\mathbf{m}^{\mathbf{3}}\right)\end{array}$ \\
\hline 40 & 0,63 & 19,69 \\
50 & 0,77 & 24,2 \\
60 & 0,92 & 28,7 \\
70 & 1,06 & 33,2 \\
\hline
\end{tabular}

Nota: $\mathrm{PMG}=$ Produção de madeira gerada a partir da colheita de $7000 \mathrm{~kg}$ de casca.

madeira produzido nos planos de manejo não interfere na escolha do plano. Isso implica que o melhor plano em resposta ao manejo sustentável, produz a lenha como um subproduto agregado ao produto de interesse. Por fim, a partir dos resultados, definiu-se os planos 40, 50, 60 e 70\% de G, são os mais sustentáveis para a produção de cascas. Baseado-se no uso atribuido a madeira pela comunidade local, esta deve ser destinada a mourões e lenha, podendo ser comercializada ou aproveitada pelo produtor rural. O manejo nas opções selecionadas deve ser aplicado dentro de um talhão em um ciclo de 10 anos, para atender a demanda de 7 toneladas de cascas, é necessário aumentar a área de abragência destes plano de manejo.

Estudos que avaliem a viabilidade econômica das opções de manejo supracitadas, devem ser realizados para definir sob este enfoque, qual a melhor opção para manejar o barbatimão no norte de Minas Gerais.

\section{CONCLUSÃO}

Conclui-se que o manejo sustentável do barbatimão no local de estudo situado no norte de Minas Gerais é viável. Para que a produção seja sustentável, a população deve ser manejada dentro dos intervalos de remoção de 40 a $70 \%$ de área basal a partir do controle da colheita pelo método GDq. A aplicação dos planos deve ter o compromisso em adotar ações que busquem o equilíbrio entre a colheita e a integridade da população para garantir a sustentabilidade do manejo.

\section{STATUS DA SUBMISSÃO}

Recebido: 29 abr., 2013

Aceito: 12 set., 2015

\section{AUTOR(ES) PARA CORRESPONDÊNCIA}

\section{Messulan Rodrigues Meira}

Programa de Pós-graduação em Ciências

Agrárias, Instituto de Ciências Agrárias, Universidade Federal de Minas Gerais - UFMG, 39404-457, Montes Claros, MG, Brasil e-mail: messulan.meira@gmail.com

\section{APOIO FINANCEIRO}

Fapemig, CNPq, Grupo Centroflora, ICA/UFMG.

\section{REFERÊNCIAS}

Almeida NA, Bittencourt AM, Santos AJ, Eisfeld CL, Souza VS. Evolução da produção e preço dos principais produtos florestais não madeireiros extrativos do Brasil. Cerne 2009; 15(3): 282-287.

Almeida SP, Proença CEB, Sano SM, Ribeiro JF. Cerrado: espécies vegetais úteis. Planaltina: Embrapa-CPAC; 1998. $464 \mathrm{p}$.

Balanço Energético Nacional - BEN. [online] 2012. [citado em 2012 nov.13]. Disponível em: http//:www.ben.epe.gov. br/ downloads/resultados_Pre_BEN_2012.pdf $<$ /eref $>$.

Borges HC Fo, Felfili JM. Avaliação dos níveis de extrativismo da casca de barbatimão Stryphnodendron adstringens (Mart.) Coville no Distrito Federal, Brasil. Revista Árvore 2003; 27(5): 735-745. http://dx.doi.org/10.1590/S010067622003000500016 .

Brasil. Coordenação de Estudos Legislativos - CEDI. Decreto $N^{o} 1.282$, de 19 de outubro de 1994. Regulamenta os artigos 15, 19, 20 e 21, da Lei $n^{\circ} 4.771$, de 15 de setembro de 1965, e dá outras providências. Diário Oficial da República Federativa do Brasil, Brasília, DF (1994 out.). [citado em 2014 jan. 10]. Disponível em: http://www2.camara.leg. br/proposicoesWeb/prop_mostrarintegra;jsessionid $=64$ E9809C9D6A8FCD3572794A468C1BB9.node2?codteor $=192751 \&$ filename $=$ LegislacaoCitada + -PL+2795/2003

British Columbia Ministry of Forests - BCMF. Introduction to silvicultural systems [online]. Victoria: BCMF; 1999. [citado em 2012 nov. 12]. Disponível em: http//www. for.gov.bc.ca/hfd/pubs/SSIntroworkbook/evenage.htm.

Camolesi, JF. Volumetria e teor alfa-bisabolol para candeia Eremanthus erythropappus [dissertação]. Lavras: 
Departamento de Ciências Florestais, Universidade Federal de Lavras; 2007. $101 \mathrm{f}$.

Côrrea VS, Cerdeira AL, Fachin AL, Bertoni BW, Pereira OS, França SC et al. Geographical variation and quality assessment of Stryphnodendron adstringens (Mart.) Coville within Brazil. Genetic Resources and Crop Evolution 2012; 59(7): 1349 - 1356.

Costa FV, Oliveira KN, Nunes YRF, Menino GCO, Brandão DO, Araújo LS et al. Florística e estrutura da comunidade arbórea de duas áreas de cerrado sentido restrito no Norte de Minas Gerais. Cerne 2010; 16(3): 267-281. http://dx.doi. org/10.1590/S0104-77602010000300003.

Faria J. Incêndios florestais: o princípio do fim. Revista da Associação de Empresas Florestais, Agrícolas e do Ambiente, ANEFA. [online] 2009 [citado em 2012 out. 22]; 5(3): 24-30. Disponível em: http//www.anefa.pt/pdf/ revista_5.pdf.

Felfili JM, Rezende RP. Conceitos e métodos em fitossociologia. Brasilia, DF: Universidade de Brasilia; 2003.

Figueiredo MAP, Souza AL, Meira JAA No, Silva AF, Figueire LHA. Alteração estrutural de uma área de cerrado explorada sob regime de manejo no município de João Pinheiro-Minas Gerais, Brasil. Revista Árvore 2010; 34(3): 521-528. http://dx.doi.org/10.1590/S010067622010000300016 .

Gama AT. Estimativas volumétricas e hipsométricas para o barbatimão no Norte de Minas Gerais como subsídio do manejo sustentado da espécie [dissertação]. Montes Claros: Instituto de Ciências Agrárias, Universidade Federal de Minas Gerais; 2012.

Gonçalves DCM, Gama JRV, Oliviera FA, Oliveira RC Jr, Araújo GCA, Almeida LS. Aspectos mercadológicos dos produtos não madeireiros na economia de Santarém-Pará, Brasil. Floresta e Ambiente 2012; 19(1): 9-16. http://dx.doi. org/10.4322/floram.2012.002.

Martins CC, Camara ATR, Machado CG, Nakagawa J. Método de superação de dormência de barbatimão. Acta Scientiarum. Agronomy 2008; 30(3): 381-385.

Meyer HA. Structure, growth, and drain in balanced uneven-aged forests. Journal of Forestry 1952; 50: 85-92.

Minas Gerais. Portaria $n^{\circ}$ 054, de 25 de agosto de 1997. Dispõe sobre Normas para Elaboração de Plano de Manejo Florestal no Estado de Minas Gerais. IEF, Belo Horizonte (1997 ago.). 19 p.

Minas Gerais. Sistema Integrado de Informação Ambiental. Lei 10561: Política Florestal no Estado de Minas Gerais. Diário do Executivo, Belo Horizonte (1991 dez.). [citado em 2012 jan. 01]. Disponível em: http://www.siam.mg.gov. br/sla/download.pdf?idNorma $=2236$
Oliveira, MC. Avaliação dos impactos de sistemas de manejo sustentável na diversidade e estrutura da flora de um Cerrado Sensu stricto [dissertação]. Lavras: Departamento de Engenharia Florestal, Universidade Federal de Lavras; 2006.

Rangel MS, Calegario N, Mello AA, Lemos PC. Melhoria na precisão da prescrição de manejo para florestal natural. Cerne 2006; 12(2): 145-156.

REMADE. Resíduos florestais para múltiplos usos. Revista da Madeira [online] 2004 [citado em 2012 out. 22]; 14(79). Disponível em: http://www.remade.com.br/ br/revistadamadeira_materia.php? num $=514 \&$ subject $=\mathrm{R}$ eciclagem\&title=Res\%EDduos\%20florestais\%20para\%20 $\mathrm{m} \%$ FAltiplos\%20usos

Ribeiro AC, Guimarães PTG, Alvarez VVH, editors. Recomendação para o uso de corretivos e fertilizantes em Minas Gerais: $5^{a}$ aproximação. Viçosa: Comissão de Fertilidade do Solo do Estado de Minas Gerais; 1999.

Rufini AL, Scolforo JRS, Oliveira AD, Mello JM. Equações volumétricas para o cerrado stricto sensu, em Minas Gerais. Cerne 2010; 16(1): 1-11. http://dx.doi.org/10.1590/ S0104-77602010000100001.

Sano SM, Almeida SP, Ribeiro JF. Cerrado: ecologia e flora. In: Sano SM, Almeida SP, Ribeiro JF. Cerrado: ecologia e flora. Brasília, DF: Embrapa Informações Tecnológicas; 2008. Cap. 1 e 2; p. 151-212.

Scolforo JRS, Oliveira AD, Davide AC. Manejo sustentado das candeias Eremanthus erythropappus (DC.) Mc Leisch e Eremanthus incanus (Less.) Less. In: Scolforo JRS, Oliveira AD, Davide AC. Manejo Sustentável da Candeia: o caminhar de uma nova experiência florestal em Minas Gerais. Lavras: UFLA; 2011.

Scolforo, SR. Manejo Florestal. Lavras: UFLA; 1998a.

Scolforo, SR. Modelagem do Crescimento e da Produção de Florestas Plantadas e Nativas. Lavras: UFLA; 1998b.

Souza AL, Oliveira MLR, Silva EF, Coelho DJS. Caracterização fitossociológica em áreas de ocorrência natural de candeia (Eremanthus erythropappus (D.C.) Mac Leish). Revista Árvore 2007; 31(4): 667-677. http://dx.doi.org/10.1590/ S0100-67622007000400011.

Souza DR, Souza AL. Emprego do método BDq de seleção após a exploração florestal em floresta ombrófila densa de terra firme, Amazônia oriental. Revista Árvore 2005; 29(4): 617-625. http://dx.doi.org/10.1590/S010067622005000400014 .

Souza FN, Scolforo JRS, Santos RM, Silva CPC. Assessment of different management systems in na area of cerrado sensu stricto. Cerne. Lavras 2011; 17(1): 85-93. 